\title{
Modified generalized marshall-olkin family of distributions
}

\author{
Muhammad Aslam ${ }^{1}$, Zawar Hussain ${ }^{1}$, Zahid Asghar ${ }^{1}$ \\ ${ }^{1}$ Department of Statistics, Quaid-i-Azam University 45320, Islamabad 44000, Pakistan \\ *Corresponding author E-mail: maslam@stat.qau.edu.pk
}

\begin{abstract}
In this article, we propose a new family of distributions using the T-X family named as modified generalized Marshall-Olkin family of distributions. Comprehensive mathematical and statistical properties of this family of distributions are provided. The model parameters are estimated by maximum likelihood method. The maximum likelihood estimation under Type-II censoring is also discussed. Two lifetime data sets are used to show the suitability and applicability of the new family of distributions. For comparison purposes, different goodness of fit tests are used.
\end{abstract}

Keywords: Burr Distribution; Maximum Likelihood Estimation; Transmuted Density; T-X Family

\section{Introduction}

Adding one or more parameters to the existing distribution or compounding one or more distribution is the key concept to generate new distributions. During the recent past, new families of probability distributions have been defined by using some well-known distributions. The purpose behind all these attempts was to provide greater flexibility, especially, to encounter the tail behavior of the distribution. This helps modelling a variety of practical data. The most important and cited distributions include the Marshall-Olkin-G family by Marshall and Olkin [13], exponentiated-G family by Gupta et al. [9], beta-G family by Eugene et al. [8], transmuted-G family by Shaw and Buckley [14], transformed-transformer-G family by Alzaatreh [1], among others. However, in many lifetime phenomena the incomplete or partial data are more attractable or reasonable instead of complete data due to sensitivity, time or cost considerations. This type of situation may be dealt with censoring methodology presented by Lawless [12], Balakrishnan \& Aggarwala [6]. Some references covering these censoring schemes can be seen in Ghitany and Al-Awadhi [10], Iliopoulos and Balakrishnan [11], Ahmad [5] and many others.

Shaw and Buckley [14] proposed a new method called transmuted maps which comprise the functional composition of cumulative density function (CDF) of one distribution with inverse CDF of the other. Its general properties were studied in Bourguinon et al. [7]. The quadratic transmutation map has the following $\mathrm{CDF}, F(x ; \lambda, \xi)=(1+\lambda) G(x ; \zeta)-\lambda G(x ; \zeta)^{2}$. It is important to see that using above transmuted CDF and taking $G(x)=x, 0<x<1$ and $\lambda=1$, we can get the CDF, $F(x)=2 x-x^{2}$. The corresponding probability density function (PDF) is given by $f(x)=2(1-x)$ which is transmuted uniform density function. Alzaatreh et al. [2] proposed a versatile methodology and named it as a T-X family. The CDF of the T-X family can be written as

$F(x ; \xi)=\int_{c}^{\omega\{G(x ; \zeta)\}} r(t ; \psi) d t$

where $r(t ; \psi)$ is a baseline PDF of a random variable $T \in[c, d]$ for $-\infty \leq c \leq d \leq \infty$ and $\omega\{G(x ; \zeta)\}$ is a function of an arbitrary CDF, which is differentiable and monotonically non-decreasing. Also, $\omega\{G(x ; \zeta)\}$ approaches to $c$ when $X$ tends to minus infinity and approaches to $d$ when $X$ tends to infinity.

Our objective, in this study, is to suggest a new family of distributions using T-X family. We take the earlier defined transmuted uniform as baseline distribution and $\omega\{G(x ; \zeta)\}=\frac{\theta G(x ; \zeta)^{\lambda}}{1+(\theta-1) G(x ; \zeta)^{\lambda}}$ in T-X family. The $\omega\{G(x ; \zeta)\}$ is obviously a generalized form of Marshall-Olkin transformation of a given exponentiated on $G(x ; \zeta)$. Thus, we have the following expression of the CDF of the proposed family of distributions.

$$
F(x ; \xi)=\frac{\theta G(x ; \zeta)^{\lambda}}{1+(\theta-1) G(x, \zeta)^{\lambda}}\left\{1+\frac{1-G(x ; \zeta)^{\lambda}}{1+(\theta-1) G(x, \zeta)^{\lambda}}\right\}
$$


where $\xi=(\theta, \lambda, \zeta)$. Shape parameter $\lambda$ coming from the exponentiation of the $G(x ; \zeta)$, scale parameter $\theta$ coming from its MarshallOlkin transformation and the parameter $\zeta$ of the baseline distribution.

The rest of the article is outlined as follows. In section 2, the probability density function of the proposed modified generalized MarshallOlkin family of distributions is definitely and its statistical property, expansions of PDF and CDF, complete and incomplete moments, moment generating function and residual and reversed residual functions are derived. In section 3 , we include estimation of parameters of the proposed family by maximum likelihood method for complete and partial data (censoring). The applications of the proposed family of distribution are discussed by considering one sub model to real data sets in section 4 . In section 5, we give the conclusion of the proposed study.

\section{The proposed probability distribution}

The PDF corresponding to CDF in (1) can be written as

$$
f(x ; \xi)=\frac{2 \lambda \theta g(x ; \zeta) G(x ; \zeta)^{\lambda-1}\left\{1-G(x ; \zeta)^{\lambda}\right\}}{\left\{1+(\theta-1) G(x, \zeta)^{\lambda}\right\}^{3}},
$$

or

$$
f(x ; \xi)=\frac{\lambda \theta g(x ; \zeta) G(x ; \zeta)^{\lambda-1}}{\left\{1+(\theta-1) G(x, \zeta)^{\lambda}\right\}^{2}}\left\{2-\frac{2 \theta G(x ; \zeta)^{\lambda}}{1+(\theta-1) G(x, \zeta)^{\lambda}}\right\},
$$

where $G(x, \zeta)$ and $g(x, \zeta)$ are arbitrary CDF and PDF of a baseline distribution, respectively. The CDF and the PDF presented in (1) and (2), respectively, are more tractable for deriving the simple and close expressions for new family of distributions using $G(x, \zeta)$ and $g(x, \zeta)$ of any baseline distribution. Hereafter, we call the variable $X$ having a density defined in (2) as the modified generalized Marshall-Olkin (MGMo) random variable.

Now, we define the reliability properties such as hazard rate and reversed hazard rate functions of the MGMo family of distributions. The hazard function $h(x ; \xi)=\frac{f(x ; \xi)}{1-F(x ; \xi)}$, using (1) and (2) is defined as

$$
h(x ; \xi)=\frac{2 \lambda \theta g(x ; \zeta) G(x ; \zeta)^{\lambda-1}\left\{1-G(x ; \zeta)^{\lambda}\right\}}{\left\{1+(\theta-1) G(x, \zeta)^{\lambda}\right\}\left[\left(1+(\theta-1) G(x, \zeta)^{\lambda}\right)^{2}-\theta G(x, \zeta)^{\lambda}\left\{2+(\theta-2) G(x, \zeta)^{\lambda}\right\}\right]},
$$

and the reversed hazard function $h^{\prime}(x ; \xi)=\frac{f(x ; \xi)}{F(x ; \xi)}$, using (1) and (2) is defined as

$h^{\prime}(x ; \xi)=\frac{2 \lambda g(x ; \zeta) G(x ; \zeta)^{-1}\left\{1-G(x ; \zeta)^{\lambda}\right\}}{2+(\theta-2) G(x, \zeta)^{\lambda}}$

The quantile function of the proposed MGMo family of distributions is given as

$$
x=G^{-1}\left[\left[\frac{1+\sqrt{1-u}}{\theta-(\theta-1)\{1+\sqrt{1-u}\}}\right]^{1 / \lambda} ; \xi\right],
$$

where, $u \in \operatorname{Uniform}(0,1)$. If we use $u=0.5$ in (3), we obtain the median. Similarly, by taking the value of $u$ between zero and one can find any quantile. Also, we can use (3) to generate random numbers from this family for simulation studies and other analyses.

\subsection{Modified generalized marshall-olkin burr distribution: sub-model}

Consider $f(x ; \alpha, \beta)=2 \alpha \beta^{2} x e^{-(\beta x)^{2}}\left[1-e^{-(\beta x)^{2}}\right]^{\alpha}$ and $F(x ; \alpha, \beta)=\left[1-e^{-(\beta x)^{2}}\right]^{\alpha}$, as the PDF and the CDF of the Burr distribution with parameters $\alpha$ and $\beta$. Then, using in (1) and (2), the CDF and the PDF of the Modified Generalized Marshall-Olkin Burr (MGMoB) distribution is obtained as 
$F(x ; \alpha, \beta, \lambda, \theta)=\frac{\theta\left[1-e^{-(\beta x)^{2}}\right]^{\alpha \lambda}}{1+(\theta-1)\left[1-e^{-(\beta x)^{2}}\right]^{\alpha \lambda}}\left\{1+\frac{1-\left[1-e^{-(\beta x)^{2}}\right]^{\alpha \lambda}}{1+(\theta-1)\left[1-e^{-(\beta x)^{2}}\right]^{\alpha \lambda}}\right\}$

And

$f(x ; \alpha, \beta, \lambda, \theta)=\frac{2 \alpha \beta^{2} \lambda \theta x e^{-(\beta x)^{2}}\left[1-e^{-(\beta x)^{2}}\right]^{\alpha-1}\left[1-e^{-(\beta x)^{2}}\right]^{\alpha(\lambda-1)}}{\left\{1+(\theta-1)\left[1-e^{-(\beta x)^{2}}\right]^{\alpha \lambda}\right\}^{2}}\left\{2-\frac{2 \theta\left[1-e^{-(\beta x)^{2}}\right]^{\alpha \lambda}}{1+(\theta-1)\left[1-e^{-(\beta x)^{2}}\right]^{\alpha \lambda}}\right\}$,

where $x>0, \theta>0, \alpha>0, \beta>0$ and $\lambda>0$.
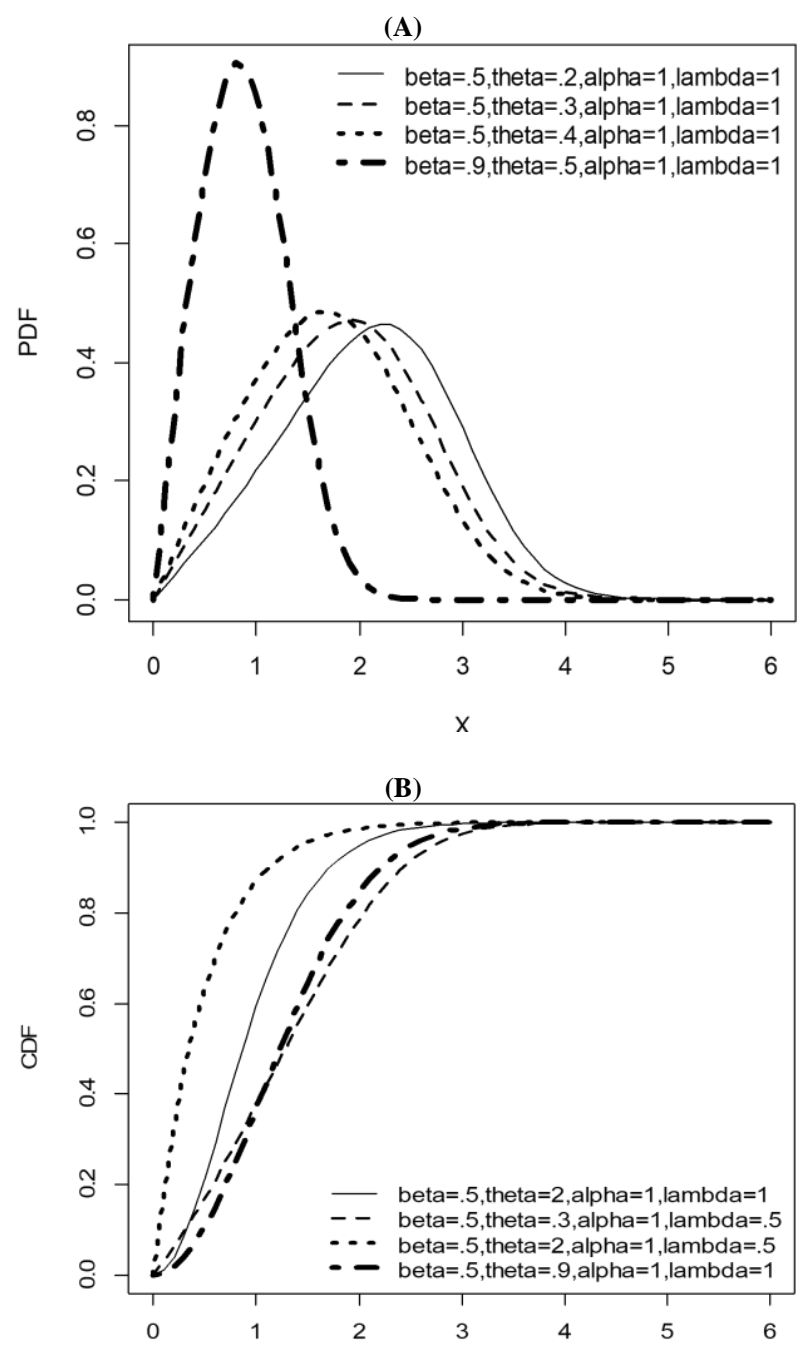

Fig. 1: Plots of PDF A) And CDF B) of the MGMoB Distribution for Different Values of Parameters.

From Fig. 1, it is obvious that the proposed MGMoB distribution is quite capable to model symmetric and skewed data. This flexibility of modelling of any type of data is actually introduced by the addition of two new parameters in the Burr distribution. Since the MGMoB distribution is a main sub-model of our proposed family, we also provide some moment expressions. In Table 1, we give first five moments $u_{n}^{\prime}, n=1,2, \ldots, 5$, the Standard Deviation (SD), Coefficient of Variation (CV), Coefficient of Skewness (CS) and Coefficient of Kurtosis (CK) for different combinations of parameters. These values are calculated via R-language.

Table 1: Moments, Standard Deviation, Coefficient of Variation, Coefficient of Skewness and Coefficient of Kurtosis of the MGMoB distribution

\begin{tabular}{llllr}
\hline$u_{n}^{\prime}$ & $\theta=0.2, \beta=0.7$, & $\theta=2, \beta=0.7$, & $\theta=2, \beta=0.7$, & $\theta=2, \beta=0.7$, \\
& $\alpha=2.5, \lambda=2$ & $\alpha=2.5, \lambda=2$ & $\alpha=0.5, \lambda=5$ & 0.8103147 \\
$u_{1}^{\prime}$ & 2.276275 & 1.598994 & 1.212651 & 0.8190424 \\
$u_{2}^{\prime}$ & 5.407719 & 2.686941 & 1.625297 & 0.5 \\
\hline
\end{tabular}




\begin{tabular}{lllll}
\hline$u_{4}^{\prime}$ & 33.90638 & 8.727994 & 3.769107 & 1.340729 \\
$u_{5}^{\prime}$ & 88.76051 & 16.81819 & 6.422578 & 2.057226 \\
SD & 0.2262914 & 0.1301589 & 0.1547761 & 0.1624325 \\
CV & 0.09941306 & 0.08140051 & 0.1276345 & 0.2004561 \\
CS & 2.882902 & 3.493977 & 3.494493 & 3.812843 \\
CK & 2.276275 & 1.598994 & 1.212651 & 0.8103147 \\
\hline
\end{tabular}

\subsection{Expansion of PDF and CDF}

We use binomial expansion to represent the PDF and CDF in expanded form. The expanded form of the PDF expressed in summation form is more conveniently used to derive the other properties of the proposed family of distributions.

The PDF in (2) can be rewritten as

$$
f(x ; \xi)=2 \lambda \theta g(x ; \zeta) G(x ; \zeta)^{\lambda-1}\left\{1+(\theta-1) G(x, \zeta)^{\lambda}\right\}^{-2}-2 \lambda \theta^{2} g(x ; \zeta) G(x ; \zeta)^{2 \lambda-1}\left\{1+(\theta-1) G(x, \zeta)^{\lambda}\right\}^{-3}
$$

Using the binomial expansion, we get

$$
\begin{aligned}
& \left\{1+(\theta-1) G(x, \zeta)^{\lambda}\right\}^{-2}=\sum_{i=0}^{\infty}\left(\begin{array}{c}
-2+i-1 \\
i
\end{array}\right)(\theta-1)^{i}(-1)^{i} G(x, \zeta)^{\lambda i}, \\
& =\sum_{i=0}^{\infty} K_{1} G(x, \zeta)^{\lambda i}, \text { where }\left(\begin{array}{c}
-2+i-1 \\
i
\end{array}\right)(\theta-1)^{i}(-1)^{i}=K_{1}, \text { and } \\
& \left\{1+(\theta-1) G(x, \zeta)^{\lambda}\right\}^{-3}=\sum_{i=0}^{\infty}\left(\begin{array}{c}
-3+i-1 \\
i
\end{array}\right)(\theta-1)^{i}(-1)^{i} G(x, \zeta)^{\lambda i}, \\
& =\sum_{i=0}^{\infty} K_{2} G(x, \zeta)^{\lambda i}, \text { where }\left(\begin{array}{c}
-3+i-1 \\
i
\end{array}\right)(\theta-1)^{i}(-1)^{i}=K_{2} .
\end{aligned}
$$

Putting above expressions in (5), we get

$$
f(x ; \xi)=2 \lambda \theta g(x ; \zeta) G(x ; \zeta)^{\lambda-1} \sum_{i=0}^{\infty} K_{1} G(x, \zeta)^{\lambda i}-2 \lambda \theta^{2} g(x ; \zeta) G(x ; \zeta)^{2 \lambda-1} \sum_{i=0}^{\infty} K_{2} G(x, \zeta)^{\lambda i}
$$

On simplification, we get

$$
f(x ; \xi)=\sum_{i=0}^{\infty} K_{1}^{*}(\lambda+\lambda i) g(x ; \zeta) G(x, \zeta)^{\lambda+\lambda i-1}-\sum_{i=0}^{\infty} K_{2}^{*}(2 \lambda+\lambda i) g(x ; \zeta) G(x, \zeta)^{2 \lambda+\lambda i-1},
$$

where $\frac{2 \theta \lambda K_{1}}{(\lambda+\lambda i)}=K_{1}^{*}$ and $\frac{2 \theta^{2} \lambda K_{2}}{(2 \lambda+\lambda i)}=K_{2}^{*}$.

Further, using exponentiated-G (exp-G) distribution, $G(x, \zeta)^{i}=H_{i}(x, \zeta)$, and

$$
h_{i+1}(x, \zeta)=\frac{d\left\{H_{i+1}(x, \zeta)\right\}}{d x}=(i+1) g(x, \zeta) G(x, \zeta)^{i}
$$

The PDF in (6) can be rewritten as

$$
f(x ; \xi)=\sum_{i=0}^{\infty}\left[K_{1}^{*} h_{\lambda+\lambda i}(x, \zeta)-K_{2}^{*} h_{2 \lambda+\lambda i}(x, \zeta)\right]
$$

Similarly, the CDF can be rewritten as

$$
F(x ; \xi)=\sum_{i=0}^{\infty}\left[K_{1}^{*} H_{\lambda+\lambda i}(x, \zeta)-K_{2}^{*} H_{2 \lambda+\lambda i}(x, \zeta)\right]
$$

Using (7) and (8), important mathematical properties, for example, the ordinary and incomplete moments and moment generating function of the proposed family of distributions can easily be derived. 


\subsection{Moments, incomplete moments and moment generating function}

If $Z_{i}$ follows exp-G distribution with power parameter i, the $K^{\text {th }}$ moment of $\mathrm{X}$, say $u_{k}^{\prime}$ can be written as

$u_{k}^{\prime}=\sum_{i=0}^{\infty}\left[K_{1}^{*} E\left\{Z_{\lambda+\lambda i}^{k}(x, \zeta)\right\}-K_{2}^{*} E\left\{Z_{2 \lambda+\lambda i}^{k}(x, \zeta)\right\}\right]$.

The $K^{\text {th }}$ central moments may be obtained as

$E\left(X-u_{1}^{\prime}\right)^{k}=\sum_{j=0}^{k}\left(\begin{array}{l}k \\ j\end{array}\right)\left(u_{1}^{\prime}\right)^{k-j} E(X)^{j}$

Putting the value of $E(X)^{j}$ from (9) in (10), we get

$E\left(X-u_{1}^{\prime}\right)^{k}=\sum_{j=0}^{k} \sum_{i=0}^{\infty}\left(\begin{array}{l}k \\ j\end{array}\right)\left(u_{1}^{\prime}\right)^{k-j}\left[K_{1}^{*} E\left\{Z_{\lambda+\lambda i}^{k}(x, \zeta)\right\}-K_{2}^{*} E\left\{Z_{2 \lambda+\lambda i}^{k}(x, \zeta)\right\}\right]$.

The moments can be obtained by putting $k=1,2, \ldots$ in (9). Thus, we can also obtain the kurtosis and skewness.

The $K^{\text {th }}$ incomplete moment of the MGMo family of distributions is defined as

$\varphi_{k}(s)=E\left(X^{k} \mid X<s\right)=\int_{-\infty}^{s} X^{k} d F(x ; \xi)$

Using the PDF given in (7), we get

$=\int_{-\infty}^{s} X^{k} \sum_{i=0}^{\infty}\left[K_{1}^{*} h_{\lambda+\lambda i}(x, \zeta)-K_{2}^{*} h_{2 \lambda+\lambda i}(x, \zeta)\right]$,

$=\sum_{i=0}^{\infty}\left[K_{1}^{*} \int_{-\infty}^{s} x^{k} h_{\lambda+\lambda i}(x, \zeta) d x-K_{2}^{*} \int_{-\infty}^{s} x^{k} h_{2 \lambda+\lambda i}(x, \zeta) d x\right]$

(12)

The first incomplete moment $\varphi_{1}(s)$ can be obtained by taking $k=1$ in (12) as

$\varphi_{1}(s)=\sum_{i=0}^{\infty}\left[K_{1}^{*} \phi_{\lambda+\lambda i}(x, \zeta)-K_{2}^{*} \phi_{2 \lambda+\lambda i}(x, \zeta)\right]$

where $\phi_{i}(s)=\int_{-\infty}^{s} x h_{i}(x, \zeta) d x$ is the $i^{\text {th }}$ incomplete moment of the exp-G distribution.

The moment generating function of the MGMo family of distributions is given in this section. Since we know that $M_{X}(t)=E\left(e^{t x}\right)$. Using (7), we can write the moment generating function (mgf) of the proposed family of distributions as follows.

$M_{X}(t)=\sum_{i=0}^{\infty}\left[K_{1}^{*} M_{\lambda+\lambda i}(t)-K_{2}^{*} M_{2 \lambda+\lambda i}(t)\right]$,

where $M_{i}(t)$ is the mgf of $Z_{i}$.

\subsection{Residual life and reversed residual life functions}

The $k^{\text {th }}$ moment of residual life function of the MGMo family of distributions is given by

$\varphi_{k}(s)=E\left((X-s)^{k} \mid X>s\right)=\frac{1}{1-F(s)} \int_{s}^{\infty}(x-s)^{k} d F(x ; \xi)$.

Using the PDF given in (7), we get

$=\frac{1}{1-F(s)} \int_{s}^{\infty}\left\{\sum_{j=0}^{k}\left(\begin{array}{l}k \\ j\end{array}\right) x^{j}(-s)^{j} \sum_{i=0}^{\infty}\left[K_{1}^{*} h_{\lambda+\lambda i}(x, \zeta)-K_{2}^{*} h_{2 \lambda+\lambda i}(x, \zeta)\right]\right\} d x$ 


$$
=\frac{1}{1-F(s)} \sum_{j=0}^{k}\left(\begin{array}{l}
k \\
j
\end{array}\right)(-s)^{j} \sum_{i=0}^{\infty}\left[K_{1}^{*} \int_{s}^{\infty} x^{j} h_{\lambda+\lambda i}(x, \zeta) d x-K_{2}^{*} \int_{s}^{\infty} x^{j} h_{2 \lambda+\lambda i}(x, \zeta) d x\right]
$$

If we put $k=1$, in (15), we obtain the mean residual life function.

Similarly, the $k^{\text {th }}$ moment of reversed residual life function can be obtained in the following way. By definition, the $k^{\text {th }}$ moment of reversed residual life function is given by

$$
\begin{aligned}
& \varphi_{k}(s)=E\left((X-s)^{k} \mid X \leq s\right), s>0, k=1,2, \ldots \\
& \varphi_{k}(s)=\frac{1}{F(s)} \int_{0}^{s}(s-x)^{k} d F(x ; \xi)
\end{aligned}
$$

Using the PDF given in (7), we get

$$
\begin{aligned}
& =\frac{1}{F(s)} \int_{0}^{s} \sum_{j=0}^{k}\left(\begin{array}{l}
k \\
j
\end{array}\right)(-x)^{j}(s)^{k-j} \sum_{i=0}^{\infty}\left[K_{1}^{*} h_{\lambda+\lambda i}(x, \zeta)-K_{2}^{*} h_{2 \lambda+\lambda i}(x, \zeta)\right], \\
& =\frac{1}{F(s)} \sum_{j=0}^{k}\left(\begin{array}{l}
k \\
j
\end{array}\right)(-1)^{j}(-s)^{k-j} \sum_{i=0}^{\infty}\left[K_{1}^{*} \int_{0}^{s} x^{j} h_{\lambda+\lambda i}(x, \zeta) d x-K_{2}^{*} \int_{0}^{s} x^{j} h_{2 \lambda+\lambda i}(x, \zeta) d x\right]
\end{aligned}
$$

If we set $k=1$ in (16), we obtain $E((s-X) \mid X \leq s)$, which is interpreted as the waiting time elapsed since failure of an item on condition that this failure had occurred in $(0, s)$.

\section{Inferential study}

Now, we present the maximum likelihood estimation of the parameters of the proposed family of distributions by using complete and partial data sets.

\subsection{Maximum likelihood estimation}

Let $x_{1}, x_{2}, \ldots, x_{n}$ are the $\mathrm{n}$ observed values of the proposed family of distributions with parameter vector $\xi$, then the likelihood function may be written as

$$
L(\xi)=\prod_{i=1}^{n}\left\{\frac{2 \lambda \theta g\left(x_{i} ; \zeta\right) G\left(x_{i} ; \zeta\right)^{\lambda-1}\left\{1-G\left(x_{i} ; \zeta\right)^{\lambda}\right\}}{\left\{1+(\theta-1) G\left(x_{i} ; \zeta\right)^{\lambda}\right\}^{3}}\right\}
$$

Taking the logarithm of the above expression, we obtain the log-likelihood function as follows

$$
l l(\xi)=n \log (2 \lambda \theta)+\sum_{i=1}^{n} \log g\left(x_{i} ; \zeta\right)+(\lambda-1) \sum_{i=1}^{n} \log G\left(x_{i} ; \zeta\right)+\sum_{i=1}^{n} \log \left\{1-G\left(x_{i} ; \zeta\right)^{\lambda}\right\}-3 \sum_{i=1}^{n} \log \left\{1+(\theta-1) G\left(x_{i} ; \zeta\right)^{\lambda}\right\}
$$

Differentiating (17) with respect to $\theta, \lambda$ and $\zeta$ and then equate to zero, we obtain the normal equations as follows

$$
\begin{aligned}
& \frac{\partial l l(\xi)}{\partial \theta}=\frac{n}{\theta}-3 \sum_{i=1}^{n}\left[\frac{G\left(x_{i} ; \zeta\right)^{\lambda}}{\left\{1+(\theta-1) G\left(x_{i} ; \zeta\right)^{\lambda}\right\}}\right]=0 \\
& \frac{\partial l l(\xi)}{\partial \lambda}=\frac{n}{\lambda}+\sum_{i=1}^{n}\left[\log G\left(x_{i} ; \zeta\right)\right]-\sum_{i=1}^{n}\left[\frac{G\left(x_{i} ; \zeta\right)^{\lambda} \log G\left(x_{i} ; \zeta\right)}{1-G\left(x_{i} ; \zeta\right)^{\lambda}}\right]-3 \sum_{i=1}^{n}\left[\frac{(\theta-1) G\left(x_{i} ; \zeta\right)^{\lambda} \log G\left(x_{i} ; \zeta\right)}{1+(\theta-1) G\left(x_{i} ; \zeta\right)^{\lambda}}\right]=0 \\
& \frac{\partial l l(\xi)}{\partial \zeta}=\sum_{i=1}^{n}\left[\frac{g^{\zeta}\left(x_{i} ; \zeta\right)}{g\left(x_{i} ; \zeta\right)}\right]+(\lambda-1) \sum_{i=1}^{n}\left[\frac{G^{\zeta}\left(x_{i} ; \zeta\right)}{G\left(x_{i} ; \zeta\right)}\right]-\lambda \sum_{i=1}^{n}\left[\frac{G\left(x_{i} ; \zeta\right)^{\lambda-1} G^{\zeta}\left(x_{i} ; \zeta\right)}{1-G\left(x_{i} ; \zeta\right)^{\lambda}}\right]-3 \lambda(\theta-1) \sum_{i=1}^{n}\left[\frac{G\left(x_{i}, \zeta\right)^{\lambda-1} G^{\zeta}\left(x_{i} ; \zeta\right)}{1+(\theta-1) G\left(x_{i} ; \zeta\right)^{\lambda}}\right]=0
\end{aligned}
$$


where $g^{\zeta}\left(x_{i} ; \zeta\right)=\frac{d g\left(x_{i} ; \zeta\right)}{d \zeta}$ and $G^{\zeta}\left(x_{i} ; \zeta\right)=\frac{d G\left(x_{i} ; \zeta\right)}{d \zeta}$. By solving nonlinear equations given in (18) to (20), numerically, the maximum likelihood estimates of the unknown parameters can be obtained. It is usually more convenient to use nonlinear optimization algorithms such as Newton-Raphson and Quasi-Raphson algorithms to find the estimates of the parameters.

\subsection{Type-II censoring}

In this sub-section, the Type-II censoring for the proposed family of distributions are discussed. Similarly, Type-I, random, progressive and Hybrid censoring may also be utilized to obtain MLEs of the parameters of the proposed family of distributions.

Let $x_{1}, x_{2}, \ldots, x_{n}$ are the $\mathrm{n}$ observed values of the proposed family of distributions. In Type-II right censoring, $t$ observations out of the $n$ are censored from the right side. The likelihood function may be written as

$$
L(\xi)=\frac{n !}{t !}\left[\prod_{i=1}^{n-t} f\left(x_{(i)} ; \xi\right)\right]\left[S\left(x_{(n-t)} ; \xi\right)\right]^{t}
$$

where $x_{(i)}$ is the order statistic of order $\mathrm{i}$, and the log-likelihood function, expressed in terms of the original baseline distribution, reads

$$
\begin{aligned}
l l(\xi)= & \ln \left(\frac{n !}{t !}\right)+(n-t) \log (2 \lambda \theta)+\sum_{i=1}^{n-t} \log g\left(x_{(i)} ; \zeta\right)+(\lambda-1) \sum_{i=1}^{n-t} \log G\left(x_{(i)} ; \zeta\right)+\sum_{i=1}^{n-t} \log \left\{1-G\left(x_{(i)} ; \zeta\right)^{\lambda}\right\}- \\
& 3 \sum_{i=1}^{n-t} \log \left\{1+(\theta-1) G\left(x_{(i)}, \zeta\right)^{\lambda}\right\}+t \log \left[1-\frac{\theta G\left(x_{(n-t)} ; \zeta\right)^{\lambda}}{1+(\theta-1) G\left(x_{(n-t)}, \zeta\right)^{\lambda}}\left\{1+\frac{1-G\left(x_{(n-t)} ; \zeta\right)^{\lambda}}{\left.1+(\theta-1) G\left(x_{(n-t)}, \zeta\right)^{\lambda}\right\}} .\right.\right.
\end{aligned}
$$

Differentiating the log-likelihood function with respect to $\theta, \lambda$ and $\zeta$, we obtain the normal equations. These normal equations can be solved analytically or numerically to find the estimates of the parameters.

\section{Applications of the MGMOB distribution}

In this section, we present the simulation study and real-life applications of the MGMoB model. We compare the sub model MGMoB of the proposed family of distributions, with Transmuted Burr (TB) distribution, Burr (BB) distribution (having two parameters), Burr (B) distribution (having one parameter), and Logistic-Weibull (LW) distributions using two real life data sets. For the ease in understanding of comparisons, we reproduce PDFs of the TB, the BB, the B and the LW distributions which are given in Appendix.

\subsection{Simulation study}

We perform Monte Carlo simulations to show the asymptotic property of the MLEs of the proposed MGMoB distribution. We calculate means, biases and mean-squared errors (MSEs) of each parameter for different sample sizes. To obtain the results, the process is replicated $\mathrm{N}=10,000$ times for $\mathrm{n}=10,20,30,50,100,200$ and 300. The simulated means, biases and MSEs are provided in the Table 2. The bias and MSE for an estimator $\hat{\theta}$ are defined as

\begin{tabular}{|c|c|c|c|c|c|}
\hline$n$ & & $\theta=2$ & $\beta=0.2$ & $\alpha=1$ & $\lambda=0.5$ \\
\hline & Mean & 4.329773 & 2.169881 & 2.384656 & 1.619396 \\
\hline \multirow[t]{3}{*}{10} & Bias & 2.329773 & 1.969881 & 1.384656 & 1.119396 \\
\hline & MSE & 5.427842 & 3.880431 & 1.917272 & 1.253047 \\
\hline & Mean & 3.448458 & 1.984185 & 1.243829 & 1.595047 \\
\hline \multirow[t]{3}{*}{20} & Bias & 1.448458 & 1.784185 & 0.243829 & 1.095047 \\
\hline & MSE & 2.098031 & 3.183316 & 0.059453 & 1.199128 \\
\hline & Mean & 2.955297 & 1.88469 & 1.212726 & 1.603641 \\
\hline \multirow{3}{*}{30} & Bias & 0.955297 & 1.68469 & 0.212726 & 1.103641 \\
\hline & MSE & 0.912592 & 2.83818 & 0.045252 & 1.218023 \\
\hline & Mean & 2.115511 & 1.890305 & 1.155103 & 1.363081 \\
\hline \multirow[t]{3}{*}{50} & Bias & 0.115511 & 1.690305 & 0.155103 & 0.863081 \\
\hline & MSE & 0.013343 & 2.857131 & 0.024057 & 0.744909 \\
\hline & Mean & 2.085921 & 1.842682 & 1.092736 & 1.048031 \\
\hline \multirow[t]{3}{*}{100} & Bias & 0.085921 & 1.642682 & 0.092736 & 0.548031 \\
\hline & MSE & 0.007382 & 2.698404 & 0.0086 & 0.300338 \\
\hline & Mean & 2.070623 & 1.89748 & 1.023095 & 0.941854 \\
\hline \multirow[t]{3}{*}{200} & Bias & 0.070623 & 1.69748 & 0.023095 & 0.441854 \\
\hline & MSE & 0.004988 & 2.881438 & 0.000533 & 0.195235 \\
\hline & Mean & 1.98498 & 1.887528 & 0.98492 & 0.625376 \\
\hline 300 & Bias & -0.01502 & 1.687528 & -0.01508 & 0.125376 \\
\hline
\end{tabular}

$$
\operatorname{Bias}(\hat{\theta})=\frac{\sum_{i=1}^{N}\left(\hat{\theta}_{i}-\theta\right)}{N} \text { and } \operatorname{MSE}(\hat{\theta})=\frac{\sum_{i=1}^{N}\left(\hat{\theta}_{i}-\theta\right)^{2}}{N}
$$

Table 2: The Simulated Means, Biases and MSEs of the MGMoB model 


\subsection{Applications using real life data sets}

In this subsection, we first describe the data sets which we have used in this study. The data 1 represent the strength measured in GPA, for single carbon fibers and impregnated 19 1000-carbon fiber tows. Single fibers were tested under tension at gauge lengths of 1, 10, 20, and $50 \mathrm{~mm}$. Impregnated tows of 1000 fibers were tested at gauge lengths of 20, 50, 150 and $300 \mathrm{~mm}$ and we are using 20mm, gauge length data. We considered the data on single fibers of $20 \mathrm{~mm}$, which is given in Appendix. The data 2 represents the life of fatigue fracture of Kevlar 373/epoxy sub- jected to constant pressure at $90 \%$ stress level until all had failed. The data 2 were previously used by Abdul-Moniem and Seham [3]. The data 2 are also given in Appendix.

The different goodness of fit tests and statistics for the MGMoB, the TB, the BB, the B, and the LW distributions are discussed. For this purpose, Anderson-Darling (A), Cramer-von Mises ( $\mathrm{W}^{*}$ ), Akaike information criterion (AIC), Bayesian information criterion (BIC), Consistent Akaike information criterion (CAIC) and Hannan-Quinn information criterion (HQIC) are considered. The general criterion in distribution theory is to choose the model as the best model among competitor models having minimum value of these statistics. The values of the $\mathrm{W}^{*}$, the A, the AIC, the CAIC, the BIC and the HQIC are calculated in R Language (using model Adequacy package) to highlight the performance of the MGMoB distribution. These results are presented in Tables 3 and 4 for data sets 1 and 2 . The Table 5 consists of values of the Kolmogorov-Smirnov test (KS) used a measure of goodness of fit for data sets 1 and 2.

Table 3: The W*, A, AIC, CAIC, BIC, HOIC for the Datal

\begin{tabular}{llllll}
\multicolumn{6}{c}{ Table 3: The $*$ A, AIC, CAIC, BIC, HQIC for the Datal } \\
\hline Model & W $^{*}$ & A & AIC & CAIC & BIC \\
MGMoB & 0.0252 & 0.2027 & 110.141 & 110.7205 & 119.357 \\
TB & NA & NA & -149.53 & -149.195 & -142.626 \\
BB & 0.1067 & 0.6923 & 112.024 & 112.193 & 116.633 \\
B & 0.0578 & 0.3868 & 190.302 & 190.357 & -146.781 \\
LW & 0.1039 & 0.6382 & 114.085 & 114.428 & 113.863 \\
\hline
\end{tabular}

Table 4: The W*, A, AIC, CAIC, BIC, HQIC for the Data2

\begin{tabular}{lllllll}
\hline Model & W $^{*}$ & A & AIC & CAIC & BIC \\
\hline MGMoB & 0.098211 & 0.583059 & 251.1683 & 251.7316 & 260.4912 \\
TB & NA & NA & 1.274775 & 250.464 & -500.7979 & -507.456 \\
BB & 0.222052 & 1.20164 & 276.6394 & 255.1674 & 259.6645 \\
B & 0.208616 & 0.907659 & 254.545 & 276.6935 & -503.259 \\
LW & 0.150799 & 254.8783 & 278.9702 & 261.5372 \\
\hline
\end{tabular}

Table 5: Kolmogorov-Smirnov (KS) Test and Probability Values

\begin{tabular}{lllll}
\hline & Data 1 & & Data 2 \\
\hline Model & KS & P-value & KS & 0.08733 \\
MGMoB & 0.0529 & 0.9857 & 2.0135 & P-value \\
TB & 4.9003 & $<2.2 \mathrm{e}-16$ & 0.15517 & $2.22 \mathrm{e}-16$ \\
BB & 0.0736 & 0.8176 & 0.20432 & 0.04598 \\
B & 0.3393 & $7.93 \mathrm{E}-08$ & 0.00293 \\
LW & 0.0588 & 0.96 & 0.08375 & 0.6300 \\
\hline
\end{tabular}

From the results in Tables 3, 4 and 5, it may be conclusively stated that the MGMoB model is a better choice for modelling the considered data sets. This finding highlights the importance of an additional parameter in the distribution. As far as, estimation of parameters is concerned, the MLEs, based on the above mentioned data sets, of all parameters for the proposed and competing distributions are arranged in the Table 6 in the Appendix.

For further demonstration, the fitted distribution and histograms of the data for the MGMoB along with other distributions are given in Fig. 2 for both data sets. The empirical CDF plots and PP-plots of the MGMoB distribution are given in Fig.s 3 and 4 for both data sets.

(A)

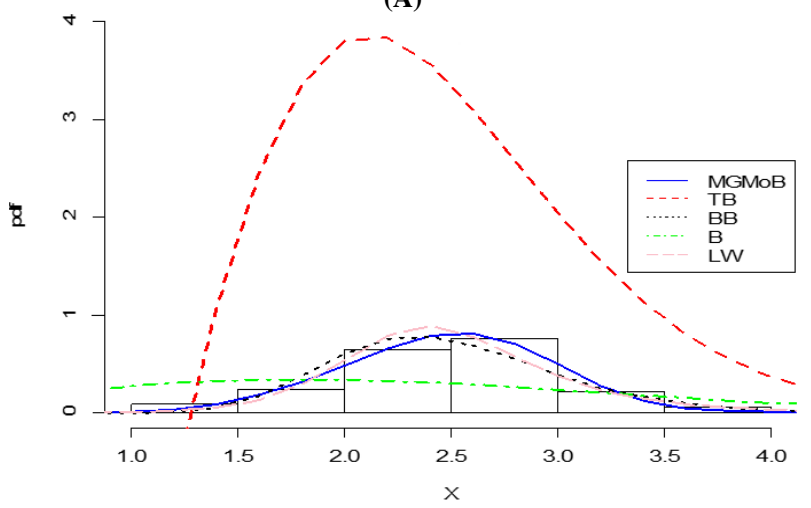


(B)

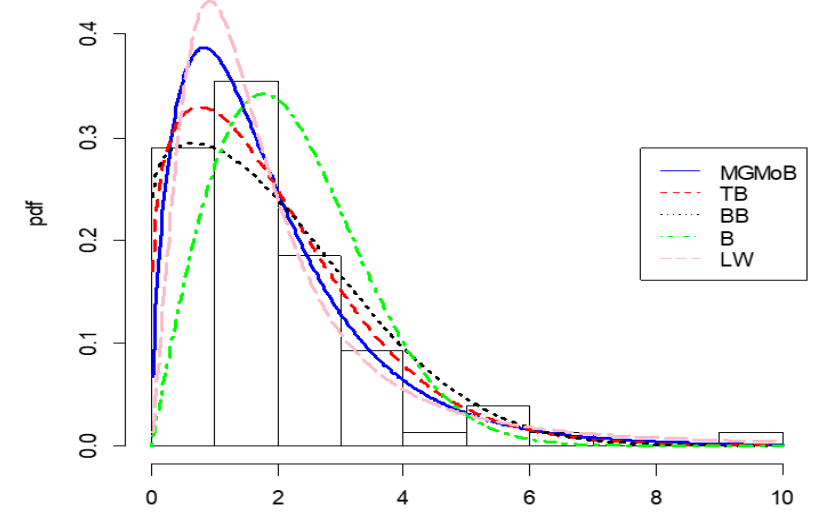

Fig. 2: Plots of Fitted Distributions and Histogram of Data 1a) and 2b).

(A)

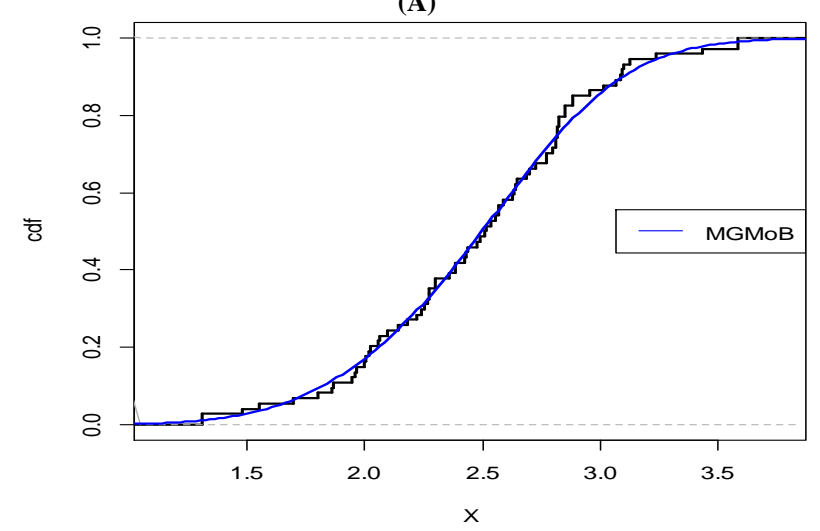

(B)

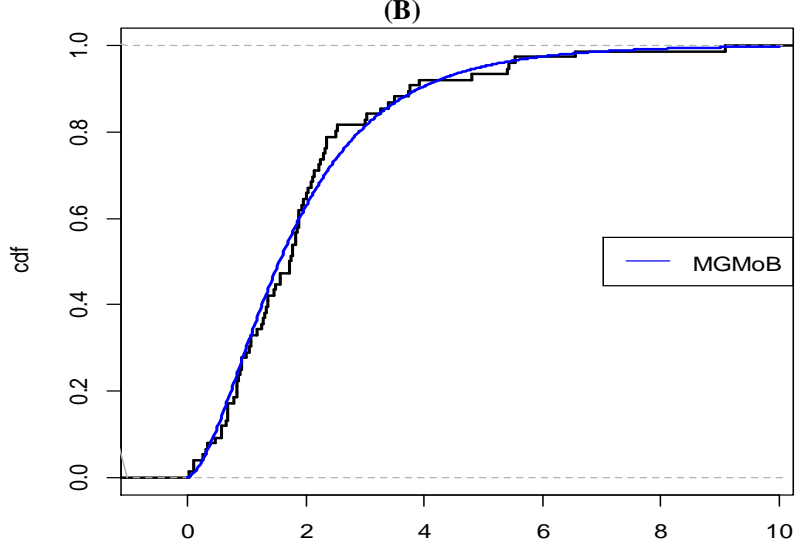

Fig. 3: Plots of Sample and Empirical CDFS of MGMOB Distribution of Data 1A) and 2B),

(A) 


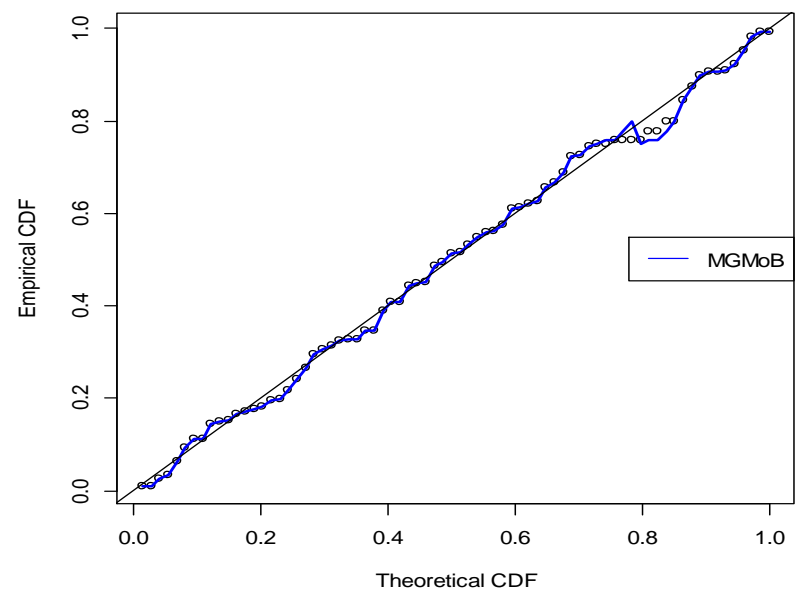

(B)

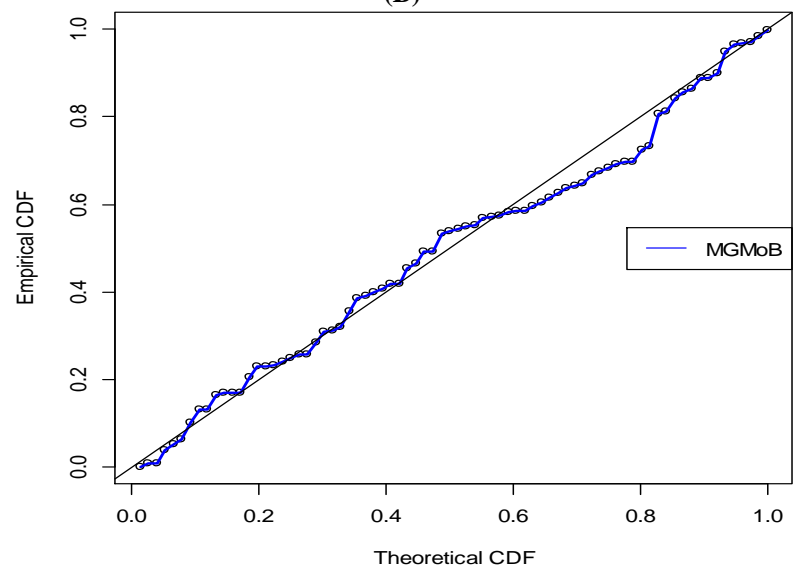

Fig. 4: PP-Plots of the MGMOB Distribution of Data 1 A) and 2 B).

\section{Conclusion}

In this article, a new family of distributions is defined by using one of the important methods called T-X family. The explicit mathematical and reliability properties of the proposed family of distributions were derived. The moments, incomplete moments and moment generatign function were simplified by using the binomial expansion. The estimation of parameters has been dealt by maximum likelihood method for complete and partial data (Type-II censoring). A special case of the proposed family using the Burr distribution has been studied, using two real lifetime data sets, to establish the appropriateness of the proposed family of distributions. The results clearly showed the better goodness of fit of the proposed model.

\section{References}

[1] Alzaatreh, A. (2011). A New Method for Generating Families of Continuous Distributions. Ph.D. Thesis, Central Michigan University, Mount Pleasant, Michigan, USA.

[2] Alzaatreh, A., Lee, C. \& Famoye, F. (2013). A new method for generating families of continuous distributions. Metron, 71, 63-79. https://doi.org/10.1007/s40300-013-0007-y.

[3] Abdul-Moniem, I. B., \& Seham, M. (2015). Transmuted gompertz distribution. Computational and Applied Mathematics Jourral, 1(3), 88-96.

[4] Alizadeh, M., Cordeiro, G. M., Nascimento, A. D., Lima, M. D. C. S., \& Ortega, E. M. (2017). Odd-Burr generalized family of distributions with some applications. Journal of Statistical Computation and Simulation, 87(2), 367-389. https://doi.org/10.1080/00949655.2016.1209200.

[5] Ahmad, Z. (2018). A New Generalized Class of Distributions: Properties and Estimation Based on Type-I Censored Samples. Annals of Data Science, 1-14. https://doi.org/10.1007/s40745-018-0160-5.

[6] Balakrishnan, N., \& Aggarwala, R. (2000). Progressive censoring: theory, methods, and applications. Springer Science \& Business Media. https://doi.org/10.1007/978-1-4612-1334-5.

[7] Bourguignon, M., Ghosh, I., \& Cordeiro, G. M. (2016). General results for the transmuted family of distributions and new models, Journal of Probability and Statistics, Vol. 2016, Article ID 7208425, 12 pages, https://doi.org/10.1155/2016/7208425.

[8] Eugene, N., Lee, C., \& Famoye, F. (2002). Beta-normal distribution and its applications. Communications in Statistics-Theory and methods, 31, 497-512. https://doi.org/10.1081/STA-120003130.

[9] Gupta, R.C., Gupta, P.I., \& Gupta, R.D. (1998). Modeling failure time data by Lehmann alternatives. Communications in Statistics-Theory and methods, 27, 887-904. https://doi.org/10.1080/03610929808832134.

[10] Ghitany, M. E., \& Al-Awadhi, S. (2002). Maximum likelihood estimation of Burr XII distribution parameters under random censoring. Journal of Applied Statistics, 29(7), 955-965. https://doi.org/10.1080/0266476022000006667.

[11] Iliopoulos, G., \& Balakrishnan, N. (2011). Exact likelihood inference for Laplace distribution based on Type-II censored samples. Journal of Statistical Planning and Inference, 141(3), 1224-1239. https://doi.org/10.1016/j.jspi.2010.09.024.

[12] Lawless, J. F. (2011). Statistical models and methods for lifetime data (Vol. 362). John Wiley \& Sons.

[13] Marshall, A.W., \& Olkin, I. (1997). A new method for adding a parameter to a family of distributions with application to the exponential and Weibull families. Biometrika, 84, 641-652. https://doi.org/10.1093/biomet/84.3.641.

[14] Shaw, W.T., Buckley, I.R.C. The alchemy of probability distributions: Beyond Gram-Charlierexpansions, and a skew kurtotic-normal distribution from a rank transmutation map. Research report, Department of Mathematics, King's College London, UK (2007). 